\title{
Allergologie
}

\section{Sind alternative Antibiotika bei Patienten mit Penicillinallergie unnötig?}

Klinikpatienten mit einer in der Akte vermerkten Penicillinallergie erhalten laut einer neuen Studie häufig alternative Antibiotika, die ihnen schaden können, obwohl diagnostische Tests zeigen, dass viele der Allergien gar nicht bestehen.

\begin{abstract}
Die Krankenhauspatienten, in deren Akte eine Penicillinallergie angegeben war, bekamen doppelt so häufig alternative Antibiotika gegen Infektionen wie Personen ohne diesen Vermerk, fanden US-amerikanische Forscher heraus. In einer früheren Untersuchung hatten sie festgestellt, dass mehr als $90 \%$ der dokumentierten Allergien nicht bestätigt werden können. „In Hinblick darauf sind die Antibiotika-Substitutionen wahrscheinlich unnötig", folgern sie.
\end{abstract}

\section{Allergieinformationen oft veraltet} Nachteilige Entscheidungen bezüglich der Antibiotikagabe kommen zustande, indem nicht überprüfte Anamnesen auf frühere Penicillinallergien der Patienten hinweisen, die inzwischen aber möglicherweise verschwunden sind, wie Forscher um Dr. Kimberly Blumenthal vom Massachusetts General Hospital in Boston erläutern.

Dadurch werden diesen Patienten häufig Alternativen zu Penicillinen und Cephalosporinen verschrieben, was ihr Risiko für unerwünschte Effekte wie nosokomiale Infektionen und An- tibiotikaresistenzen erhöhen könne, so Blumenthal und Kollegen. Das ließe sich in vielen Fällen verhindern, indem Ärzte mehr testen oder mit den Patienten über die vermeintliche Allergie sprechen, statt die Information einfach ungeprüft zu übernehmen.

Die Forscher analysierten Daten von fast 11.000 Patienten aus 106 USamerikanischen Krankenhäusern. Sie stellten fest, dass diejenigen mit einer dokumentierten Penicillinallergie (16\%) signifikant häufiger mit einer Alternative zu $\beta$-Lactam-Antibiotika behandelt wurden, etwa mit Clindamycin, Linezolid, Fluorchinolonen, Aminoglykosiden, Tetrazyklinen oder Vancomycin. Diese Patienten erhielten auch fünfmal häufiger das mit Clostridium-difficile-Infektionen assoziierte Clindamycin als diejenigen ohne Allergievermerk.

\section{Vorsicht bei perioperativer Antibiose}

Stationäre Patienten mit dokumentierter Penicillinallergie, die Antibiotika als Prophylaxe bei einer bevorstehenden Operation benötigten, erhielten siebenmal so häufig ein alter-
Quelle:

Joana Schmidt,

SpringerMedizin.de

\section{Das Wichtigste in Kürze}

Wie wirken sich in Krankenakten vermerkte Penicillinallergien bei Klinikpatienten auf die Antibiotikagabe aus?

Oft werden alternative Antibiotika eingesetzt, die schaden können, obwohl die Allergien nicht mehr bestehen.

Bedeutung: Bei einer dokumentierten Penicillinallergie sollte durch Tests oder Patientengespräche geprüft werden, ob eine solche Allergie wirklich vorliegt.

Einschränkung: Da Daten aus US-amerikanischen Krankenhäusern verwendet wurden, sind die Ergebnisse möglicherweise nicht international repräsentativ. natives Antibiotikum, obwohl für diese Indikation $\beta$-Lactam-Antibiotika empfohlen werden. „Ärzte sollten besonders darauf achten, potenzielle Allergien gegen Penicillin bei Patienten richtig zu beurteilen, bei denen chirurgische Eingriffe geplant sind und bei denjenigen, die sonst Clindamycin bekommen würden", raten die Forscher.

Zwar gebe es einen diagnostischen Test, der bei der Einschätzung helfe, doch fehle vielen Krankenhäusern der Zugang dazu, bemängeln Blumenthal und Kollegen. Ihr Fazit: Ein aufmerksames und proaktives Vorgehen sei wünschenswert, da das Verschreiben von Antibiotika ohne vollständige Informationen über mögliche Penicillinallergien den Patienten letztendlich mehr schaden als nützen könne.

\section{Literatur \\ 1. Blumenthal KG et al (2020) Association Bet- ween Penicillin Allergy Documentation and Antibiotic Use. JAMA INtern Med. https:// doi.org/10.1001/jamainternmed.2020.2227}

Hinweis des Verlags. Der Verlag bleibt in Hinblick auf geografische Zuordnungen und Gebietsbezeichnungen in veröffentlichten Karten und Institutsadressen neutral.

hautnah $2020 \cdot 19: 140$ https://doi.org/10.1007/s12326-02000398-3

(c) Springer-Verlag GmbH Austria, ein Teil von Springer Nature 2020 